Research Article

\title{
Boundedness of Commutators of High-Dimensional Hausdorff Operators
}

\author{
Guilian Gao and Houyu Jia \\ Department of Mathematics, Zhejiang University, Hangzhou 310027, China \\ Correspondence should be addressed to Houyu Jia, mjhy@zju.edu.cn \\ Received 15 February 2012; Accepted 16 March 2012 \\ Academic Editor: Dashan Fan
}

Copyright (C) 2012 G. Gao and H. Jia. This is an open access article distributed under the Creative Commons Attribution License, which permits unrestricted use, distribution, and reproduction in any medium, provided the original work is properly cited.

We prove the boundedness of commutators of high-dimensional Hausdorff operators $H_{\Phi, b}$ on Lebesgue space with central BMO function or Lipschitz function. Furthermore, the boundedness on Herz spaces and Morrey-Herz spaces is also obtained.

\section{Introduction}

The one-dimensional Hausdorff operator is defined as

$$
h_{\Phi} f(x)=\int_{0}^{\infty} \frac{\Phi(t)}{t} f\left(\frac{x}{t}\right) d t
$$

where $\Phi$ is a locally integrable function on $(0, \infty)$. The operator $h_{\Phi}$ has a deep root in the study of the one-dimensional Fourier analysis. Particularly, it is closely related to the summability of the classical Fourier series. The reader can see ([1-3]) to find details of background and recent development of the Hausdorff operator.

Recently, Hausdorff operator in the high dimensional space $\mathbb{R}^{n}$ was studied. Three extensions of the one-dimensional Hausdorff operator in $\mathbb{R}^{n}$ were recently introduced and studied in [4]. One of them is the operator

$$
H_{\Phi} f(x)=\int_{\mathbb{R}^{n}} \frac{\Phi\left(|y|^{-1} x\right)}{|y|^{n}} f(y) d y,
$$


where $\Phi$ is a radial function defined on $\mathbb{R}^{+}$. Replacing $\Phi$ with

$$
\Phi_{1}(t)=t^{-n} \chi_{(1, \infty)}(t), \quad \Phi_{2}(t)=\chi_{(0,1)}(t),
$$

$H_{\Phi} f$ becomes the high dimensional Hardy operator

$$
H f(x)=\frac{1}{|x|^{n}} \int_{|y|<|x|} f(y) d y, \quad x \in \mathbb{R}^{n} \backslash\{0\},
$$

and its adjoint operator

$$
H^{*} f(x)=\int_{|y| \geq|x|} \frac{f(y)}{|y|^{n}} d y
$$

respectively. It is well known that Hardy operators are important operators in Harmonic analysis and a quite number of papers have appeared in [5-9]. And in [10], the authors have obtained the boundedness of commutators of one-dimensional Hausdorff operator with onesided dyadic $\mathrm{CMO}$ functions on the Lebesgue space.

These observations motivate us to study the boundedness of commutators of $n$-dimensional Hausdorff operators on some function spaces. Let $b(x)$ be a real measurable, locally integrable function; we define the commutators of Hausdorff operators as follows:

$$
\begin{aligned}
H_{\Phi, b} f(x)= & b(x) H_{\Phi} f(x)-H_{\Phi}(b f)(x)=\int_{\mathbb{R}^{n}} \frac{\Phi\left(|y|^{-1} x\right)}{|y|^{n}}(b(x)-b(y)) f(y) d y \\
= & \int_{|y|<|x|} \frac{\Phi\left(|y|^{-1} x\right)}{|y|^{n}}(b(x)-b(y)) f(y) d y \\
& +\int_{|y| \geq|x|} \frac{\Phi\left(|y|^{-1} x\right)}{|y|^{n}}(b(x)-b(y)) f(y) d y \\
:= & H_{\Phi, b}^{1} f(x)+H_{\Phi, b}^{2} f(x) .
\end{aligned}
$$

Definition 1.1 (see [11]). Let $1<q<\infty$. A function $f \in L_{\text {loc }}^{q}\left(R^{n}\right)$ is said to belong to the space $\mathrm{CMO}^{q}\left(\mathbb{R}^{n}\right)$ if

$$
\|f\|_{\mathrm{CMO}^{q}\left(\mathbb{R}^{n}\right)}=\sup _{r>0}\left(\frac{1}{|B(0, r)|} \int_{B(0, r)}\left|f(x)-f_{B}\right|^{q} d x\right)^{1 / q}<\infty,
$$

where $f_{B}=(1 /|B(0, r)|) \int_{B(0, r)} f(x) d x$. Obviously, we have $\mathrm{BMO}\left(\mathbb{R}^{n}\right) \subset \mathrm{CMO}^{q}\left(\mathbb{R}^{n}\right)$ for all $1 \leq q<\infty$, and $\mathrm{CMO}^{q}\left(\mathbb{R}^{n}\right) \subset \mathrm{CMO}^{p}\left(\mathbb{R}^{n}\right), 1 \leq p<q<\infty$. 
Definition 1.2 (see [12]). Let $0<\gamma<1$. The Besov-Lipschitz space $\dot{\Lambda}_{\gamma}\left(\mathbb{R}^{n}\right)$ is defined by

$$
\|b\|_{\Lambda_{r}\left(\mathbb{R}^{n}\right)}=\sup _{x, h \in \mathbb{R}^{n}} \frac{|b(x+h)-b(x)|}{|h|^{\gamma}}<\infty .
$$

Herz-type spaces are important function spaces in harmonic analysis. It should be pointed out that $\mathrm{Lu}$ and Yang make tremendous contributions on this spaces. Their book (joint with $\mathrm{Hu}$ ) [13] is the unique research book in this topic. Below, we briefly recall the definition of the Herz-type spaces. We denote by $B(x, r)$ the ball centered at $x$ with radius $r$. $C$ is a constant which may vary from line to line. For $k \in \mathbb{Z}$, let $B_{k}=\left\{x \in \mathbb{R}^{n}:|x| \leq 2^{k}\right\}$, $\Delta_{k}=B_{k} \backslash B_{k-1}$, and $\chi_{k}(k \in \mathbb{Z})$ denote the characteristic function of the set $\Delta_{k}$.

Definition 1.3 (see [13]). Let $\alpha \in \mathbb{R}, 0<p<\infty$, and $0<q<\infty$. The homogeneous Herz space $\dot{K}_{q}^{\alpha, p}\left(\mathbb{R}^{n}\right)$ is defined by

$$
\dot{K}_{q}^{\alpha, p}\left(\mathbb{R}^{n}\right)=\left\{f \in L_{\mathrm{loc}}^{q}\left(\mathbb{R}^{n} \backslash\{0\}\right):\|f\|_{\dot{K}_{q}^{\alpha, p}\left(\mathbb{R}^{n}\right)}<\infty\right\},
$$

where

$$
\|f\|_{\dot{K}_{q}^{\alpha, p}\left(\mathbb{R}^{n}\right)}=\left(\sum_{k=-\infty}^{\infty} 2^{k \alpha p}\left\|f_{X_{k}}\right\|_{L^{q}\left(\mathbb{R}^{n}\right)}^{p}\right)^{1 / p} .
$$

Obviously, $\dot{K}_{q}^{0, q}\left(\mathbb{R}^{n}\right)=L^{q}\left(\mathbb{R}^{n}\right)$ and $\dot{K}_{q}^{\alpha / q, q}\left(\mathbb{R}^{n}\right)=L^{q}\left(\mathbb{R}^{n},|x|^{\alpha}\right)$, so the Herz space is the natural generalization of the Lebesgue spaces with power weight $|x|^{\alpha}$.

Definition 1.4 (see [13]). Let $\alpha \in \mathbb{R}, 0<p \leq \infty, 0<q<\infty$ and $\lambda \geq 0$. The homogeneous Morrey-Herz space $M \dot{K}_{p, q}^{\alpha, \lambda}\left(\mathbb{R}^{n}\right)$ is defined by

$$
M \dot{K}_{p, q}^{\alpha, \lambda}\left(\mathbb{R}^{n}\right)=\left\{f \in L_{\mathrm{loc}}^{q}\left(\mathbb{R}^{n} \backslash\{0\}\right):\|f\|_{M \dot{K}_{p, q}^{\alpha, \lambda}\left(\mathbb{R}^{n}\right)}<\infty\right\},
$$

where

$$
\|f\|_{M \dot{K}_{p, q}^{\alpha, \lambda}\left(\mathbb{R}^{n}\right)}=\sup _{k_{0} \in \mathbb{Z}} 2^{-k_{0} \lambda}\left(\sum_{k=-\infty}^{k_{0}} 2^{k \alpha p}\left\|f \chi_{k}\right\|_{L^{q}\left(\mathbb{R}^{n}\right)}^{p}\right)^{1 / p}
$$

with the usual modification made when $p=\infty$.

In [14], the Morrey space $M_{q}^{\lambda}\left(\mathbb{R}^{n}\right)$ is defined by

$$
M_{q}^{\lambda}\left(\mathbb{R}^{n}\right)=\left\{f \in L_{\mathrm{loc}}^{q}\left(\mathbb{R}^{n}\right): \sup _{\lambda>0, x \in \mathbb{R}^{n}} \frac{1}{r^{\lambda}} \int_{|x-y|<r}|f(y)|^{q} d y<\infty\right\} .
$$

Obviously, $M \dot{K}_{p, q}^{\alpha, 0}\left(\mathbb{R}^{n}\right)=\dot{K}_{q}^{\alpha, p}\left(\mathbb{R}^{n}\right)$ and $M_{q}^{\lambda}\left(\mathbb{R}^{n}\right) \subset M \dot{K}_{q, q}^{0, \lambda}\left(\mathbb{R}^{n}\right)$. 


\section{Main Results}

Now, we state our main results.

Theorem 2.1. Let $1<p<\infty, 1<r<\min \left(p, p^{\prime}\right)$, and $b \in C \dot{M} O^{\max (p, p r /(p-r))}\left(\mathbb{R}^{n}\right)$ :

(a) if one sets

$$
A_{\Phi, r}=\left(\int_{0}^{\infty}|\Phi(t)|^{r^{\prime}} t^{-1+n\left(r^{\prime} / r\right)} d t\right)^{1 / r^{\prime}}<\infty
$$

then $H_{\Phi, b}^{1}$ is bounded on $L^{p}\left(\mathbb{R}^{n}\right)$;

(b) if one sets

$$
B_{\Phi, r}=\left(\int_{0}^{\infty}|\Phi(t)|^{r^{\prime}} t^{-1+n} d t\right)^{1 / r^{\prime}}<\infty
$$

then $H_{\Phi, b}^{2}$ is bounded on $L^{p}\left(\mathbb{R}^{n}\right)$;

(c) if $\Phi$ satisfies (2.1) and (2.2), then $H_{\Phi, b}$ is bounded on $L^{p}\left(\mathbb{R}^{n}\right)$.

Remark 2.2. When $\Phi(t)=t^{-n} X_{(1, \infty)}(t), H_{\Phi, b} f(x)=H_{\Phi, b}^{1} f(x)=H_{b} f(x)$. We can check that $\Phi(t)$ satisfies (2.1); therefore, the boundedness of commutator of Hardy operator is obtained. When $\Phi(t)=\chi_{(0,1)}(t), H_{\Phi, b} f(x)=H_{\Phi, b}^{2} f(x)=H_{b}^{*} f(x)$. We also know that $\Phi(t)$ satisfies (2.2); therefore, we get the boundedness of commutator of the adjoint Hardy operator see [9].

Remark 2.3. Using the same method, we can get the generalized result of Theorem 2.1(b). Let $1<p<\infty, 1<r<p, 0 \leq \lambda<\min \left(r^{\prime} / r, r^{\prime} / p\right)$, and $b \in \mathrm{CMO}^{\max (p, p r /(p-r))}\left(\mathbb{R}^{n}\right)$. If

$$
B_{\Phi, r, \lambda}=\left(\int_{0}^{\infty}|\Phi(t)|^{r^{\prime}} t^{-1+n \lambda} d t\right)^{1 / r^{\prime}}<\infty,
$$

then $H_{\Phi, b}^{2}$ is bounded on $L^{p}\left(\mathbb{R}^{n}\right)$.

Theorem 2.4. Let $1<p, q<\infty, 1<r<\min \left(p, q^{\prime}\right), 1 / p-1 / q=\gamma / n$, and $b \in \dot{\Lambda}_{\gamma}\left(\mathbb{R}^{n}\right)(0<\gamma<$ 1). Then

(a) if $\Phi$ satisfies (2.1), then $H_{\Phi, b}^{1}$ are bounded from $L^{p}\left(\mathbb{R}^{n}\right)$ to $L^{q}\left(\mathbb{R}^{n}\right)$;

(b) if $\Phi$ satisfies (2.2), then $H_{\Phi, b}^{2}$ are bounded from $L^{p}\left(\mathbb{R}^{n}\right)$ to $L^{q}\left(\mathbb{R}^{n}\right)$;

(c) if $\Phi$ satisfies (2.1) and (2.2), then $H_{\Phi, b}$ is bounded from $L^{p}\left(\mathbb{R}^{n}\right)$ to $L^{q}\left(\mathbb{R}^{n}\right)$.

Remark 2.5. Just like in Remark 2.2, we get Lipschitz estimates for commutator of Hardy operator or the adjoint Hardy operator. See the details in [15].

Theorem 2.6. Let $0<p<\infty, 1<q<\infty, 1<r<q$, and $b \in C \dot{M} O^{\max (q, q r /(q-r))}\left(\mathbb{R}^{n}\right)$. Then

(a) if $\Phi$ satisfies (2.1) and $\alpha<n(1 / r-1 / q)$, then $H_{\Phi, b}^{1}$ is bounded on $\dot{K}_{q}^{\alpha, p}\left(\mathbb{R}^{n}\right)$;

(b) if $\Phi$ satisfies (2.2) and $\alpha>-n\left(1 / q-1 / r^{\prime}\right)$, then $H_{\Phi, b}^{2}$ is bounded on $\dot{K}_{q}^{\alpha, p}\left(\mathbb{R}^{n}\right)$;

(c) if $\Phi$ satisfies (2.1), (2.2), and $-n\left(1 / q-1 / r^{\prime}\right)<\alpha<n(1 / r-1 / q)$, then $H_{\Phi, b}$ is bounded on $\dot{K}_{q}^{\alpha, p}\left(\mathbb{R}^{n}\right)$.

Remark 2.7. Let $\alpha=0$ in Theorem 2.6, then Theorem 2.1 can be obtained. 
Journal of Function Spaces and Applications

Theorem 2.8. Let $0<p_{1} \leq p_{2}<\infty, 1<q_{1}, q_{2}<\infty, 1<r<q_{1}, 1 / q_{1}-1 / q_{2}=r / n$, and $b \in \Lambda_{\gamma}\left(\mathbb{R}^{n}\right)(0<\gamma<1)$. Then

(a) if $\Phi$ satisfies (2.1) and $\alpha<n\left(1 / r-1 / q_{1}\right)$, then $H_{\Phi, b}^{1}$ is bounded from $\dot{K}_{q_{1}}^{\alpha, p_{1}}\left(\mathbb{R}^{n}\right)$ to $\dot{K}_{q_{2}}^{\alpha, p_{2}}\left(\mathbb{R}^{n}\right)$;

(b) if $\Phi$ satisfies (2.2) and $\alpha>-n\left(1 / q_{2}-1 / r^{\prime}\right)$, then $H_{\Phi, b}^{2}$ are bounded from $\dot{K}_{q_{1}}^{\alpha, p_{1}}\left(\mathbb{R}^{n}\right)$ to $\dot{K}_{q_{2}}^{\alpha, p_{2}}\left(\mathbb{R}^{n}\right)$;

(c) if $\Phi$ satisfies (2.1), (2.2), and $-n\left(1 / q_{2}-1 / r^{\prime}\right)<\alpha<n\left(1 / r-1 / q_{1}\right)$, then $H_{\Phi, b}$ is bounded from $\dot{K}_{q_{1}}^{\alpha, p_{1}}\left(\mathbb{R}^{n}\right)$ to $\dot{K}_{q_{2}}^{\alpha, p_{2}}\left(\mathbb{R}^{n}\right)$.

Remark 2.9. Let $\alpha=0$ in Theorem 2.8, then Theorem 2.4 can be obtained.

Theorem 2.10. Let $0<p<\infty, 1<q<\infty, 1<r<q, \lambda \geq 0$, and $b \in C \dot{M} O^{\max (q, q r /(q-r))}\left(\mathbb{R}^{n}\right)$. Then

(a) if $\Phi$ satisfies (2.1) and $\alpha<n(1 / r-1 / q)+\lambda$, then $H_{\Phi, b}^{1}$ is bounded on $M \dot{K}_{p, q}^{\alpha, \lambda}\left(\mathbb{R}^{n}\right)$;

(b) if $\Phi$ satisfies (2.2) and $\alpha>-n\left(1 / q-1 / r^{\prime}\right)+\lambda$, then $H_{\Phi, b}^{2}$ is bounded on $M \dot{K}_{p, q}^{\alpha, \lambda}\left(\mathbb{R}^{n}\right)$;

(c) if $\Phi$ satisfies (2.1), (2.2), and $-n\left(1 / q-1 / r^{\prime}\right)+\lambda<\alpha<n(1 / r-1 / q)+\lambda$, then $H_{\Phi, b}$ is bounded on $M \dot{K}_{p, q}^{\alpha, \lambda}\left(\mathbb{R}^{n}\right)$.

Theorem 2.11. Let $\lambda \geq 0,0<p_{1} \leq p_{2}<\infty, 1<q_{1}, q_{2}<\infty, 1<r<q_{1}, 1 / q_{1}-1 / q_{2}=\gamma / n$, and $b \in \dot{\Lambda}_{\gamma}\left(\mathbb{R}^{n}\right)(0<\gamma<1)$.

(a) if $\Phi$ satisfies (2.1) and $\alpha<n\left(1 / r-1 / q_{1}\right)+\lambda$, then $H_{\Phi, b}^{1}$ is bounded from $M \dot{K}_{p_{1}, q_{1}}^{\alpha, \lambda}\left(\mathbb{R}^{n}\right)$ to $M \dot{K}_{p_{2}, q_{2}}^{\alpha, \lambda}\left(\mathbb{R}^{n}\right)$;

(b) if $\Phi$ satisfies (2.2) and $\alpha>-n\left(1 / q_{2}-1 / r^{\prime}\right)+\lambda$, then $H_{\Phi, b}^{2}$ is bounded from $M \dot{K}_{p_{1}, q_{1}}^{\alpha, \lambda}\left(\mathbb{R}^{n}\right)$ to $M \dot{K}_{p_{2}, q_{2}}^{\alpha, \lambda}\left(\mathbb{R}^{n}\right)$;

(c) if $\Phi$ satisfies (2.1), (2.2), and $-n\left(1 / q_{2}-1 / r^{\prime}\right)+\lambda<\alpha<n\left(1 / r-1 / q_{1}\right)+\lambda$, then $H_{\Phi, b}$ is bounded from $M \dot{K}_{p_{1}, q_{1}}^{\alpha, \lambda}\left(\mathbb{R}^{n}\right)$ to $M \dot{K}_{p_{2}, q_{2}}^{\alpha, \lambda}\left(\mathbb{R}^{n}\right)$.

\section{Proof of the Main Results}

We first give several lemmas.

Lemma 3.1. Let $1<r \leq p<\infty$ and $\lambda \geq 0$, then

(i) $\int_{\Delta_{i}}\left(|\Phi(x /|y|)| /|y|^{n}\right)|f(y)| d y \leq C A_{\Phi, r} 2^{i n(1 / r-1 / p)}|x|^{-n / r}\left\|f X_{i}\right\|_{L^{p}\left(\mathbb{R}^{n}\right)^{\prime}}$

(ii) $\int_{\Delta_{i}}\left(|\Phi(x /|y|)| /|y|^{n}\right)|f(y)| d y \leq C B_{\Phi, r, \lambda} 2^{i n\left(\lambda / r^{\prime}-1 / p\right)}|x|^{-n \lambda / r^{\prime}}\left\|f X_{i}\right\|_{L^{p}\left(\mathbb{R}^{n}\right)}$. 
Proof. (i) By Hölder's inequality and polar coordinate, we obtain

$$
\begin{aligned}
& \int_{\Delta_{i}} \frac{|\Phi(x /|y|)|}{|y|^{n}}|f(y)| d y \leq\left(\int_{\Delta_{i}} \frac{|\Phi(x /|y|)|^{r^{\prime}}}{|y|^{n r^{\prime}}} d y\right)^{1 / r^{\prime}}\left(\int_{\Delta_{i}}|f(y)|^{r} d y\right)^{1 / r} \\
& \leq\left(\int_{|x| / 2^{i}}^{|x| / 2^{i-1}}\left|S^{n-1}\right||\Phi(t)|^{r^{\prime}} t^{-1+n r^{\prime} / r}|x|^{n\left(1-r^{\prime}\right)} d t\right)^{1 / r^{\prime}} \\
& \times\left(\int_{\Delta_{i}}|f(y)|^{p} d y\right)^{1 / p} 2^{i n(1 / r-1 / p)} \\
& \leq C A_{\Phi, r} 2^{i n(1 / r-1 / p)}|x|^{-n / r}\left\|f X_{i}\right\|_{L^{p}\left(\mathbb{R}^{n}\right)} .
\end{aligned}
$$

(ii) By Hölder's inequality and polar coordinate, we have

$$
\begin{aligned}
\int_{\Delta_{i}} \frac{|\Phi(x /|y|)|}{|y|^{n}}|f(y)| d y \leq & \left(\int_{\Delta_{i}} \frac{|\Phi(x /|y|)|^{r^{\prime}}}{|y|^{\left(n(\lambda+1) / r^{\prime}\right) r^{\prime}}} d y\right)^{1 / r^{\prime}}\left(\int_{\Delta_{i}} \frac{|f(y)|^{r}}{|y|^{\left(n-n(\lambda+1) / r^{\prime}\right) r}} d y\right)^{1 / r} \\
\leq & C\left(\int_{2^{i-1}}^{2^{i}} \int_{S^{n-1}}\left|\Phi\left(|x| R^{-1}\right)\right|^{r^{\prime}} R^{-1-n \lambda} d \sigma\left(y^{\prime}\right) d R\right)^{1 / r^{\prime}} \\
& \times 2^{-i n\left(1-(\lambda+1) / r^{\prime}\right)}\left\|f X_{i}\right\|_{L^{r}} \\
\leq & C\left(\int_{2^{i-1}}^{2^{i}} \int_{S^{n-1}}\left|\Phi\left(|x| R^{-1}\right)\right|^{r^{\prime}} R^{-1-n \lambda} d \sigma\left(y^{\prime}\right) d R\right)^{1 / r^{\prime}} \\
& \times 2^{i n\left(\lambda / r^{\prime}-1 / p\right)}\left\|f X_{i}\right\|_{L^{p}} \\
\leq & C B_{\Phi, r, \lambda} 2^{i n\left(\lambda / r^{\prime}-1 / p\right)}|x|^{-n \lambda / r^{\prime}}\left\|f X_{X_{i}}\right\|_{L^{p}} .
\end{aligned}
$$

Lemma 3.2 (see [9]). Let b be a $C \dot{M} O^{1}\left(\mathbb{R}^{n}\right)$ function and $i, k \in \mathbb{Z}$, then

$$
\left|b(t)-b_{B_{i}}\right| \leq\left|b(t)-b_{B_{k}}\right|+2|i-k|\|b\|_{C \dot{M} O^{1}\left(\mathbb{R}^{n}\right)} .
$$

Lemma 3.3 (see [12]). Let $0<\gamma<1$ and $f \in \dot{\Lambda}_{\gamma}\left(\mathbb{R}^{n}\right)$. Then

$$
|f(x)-f(y)| \leq|x-y|^{\gamma}\|f\|_{\Lambda_{\gamma}\left(\mathbb{R}^{n}\right)} \leq\left(|x|^{\gamma}+|y|^{\gamma}\right)\|f\|_{\Lambda_{\gamma}\left(\mathbb{R}^{n}\right)} .
$$

Proof of Theorem 2.6. (a) When $\Phi$ satisfies (2.1) and $\alpha<n(1 / r-1 / q)$, we get

$$
\begin{aligned}
\left\|\left(H_{\Phi, b}^{1} f\right) x_{k}\right\|_{L^{q}}^{q} \leq & C_{q} \int_{\Delta_{k}}\left(\sum_{i=-\infty}^{k} \int_{\Delta_{i}} \frac{|\Phi(x /|y|)|}{|y|^{n}}\left|\left(b(x)-b_{B_{k}}\right) f(y)\right| d y\right)^{q} d x \\
& +C_{q} \int_{\Delta_{k}}\left(\sum_{i=-\infty}^{k} \int_{\Delta_{i}} \frac{|\Phi(x /|y|)|}{|y|^{n}}\left|\left(b(y)-b_{B_{k}}\right) f(y)\right| d y\right)^{q} d x \\
:= & I+I I .
\end{aligned}
$$


For I, using Lemma 3.1(i), we have

$$
\begin{aligned}
I & \leq C_{q} A_{\Phi, r}^{q} \int_{B_{k}}\left|b(x)-b_{B_{k}}\right|^{q}\left(\sum_{i=-\infty}^{k} 2^{i n(1 / r-1 / q)}|x|^{-n / r}\left\|f X_{i}\right\|_{L^{q}}\right)^{q} d x \\
& \leq C_{q} A_{\Phi, r}^{q}\|b\|_{\mathrm{CMO}^{q}\left(\mathbb{R}^{n}\right)}^{q}\left|B_{k}\right|\left(\sum_{i=-\infty}^{k} 2^{i n(1 / r-1 / q)} 2^{-k n / r}\left\|f X_{i}\right\|_{L^{q}}\right)^{q} \\
& =C_{q} A_{\Phi, r}^{q}\|b\|_{\mathrm{CMO}^{q}\left(\mathbb{R}^{n}\right)}^{q}\left(\sum_{i=-\infty}^{k} 2^{(i-k) n(1 / r-1 / q)}\left\|f X_{i}\right\|_{L^{q}}\right)^{q} .
\end{aligned}
$$

For II, by Lemma 3.2, we have

$$
\begin{aligned}
I I \leq & C_{q} \int_{\Delta_{k}}\left(\sum_{i=-\infty}^{k} \int_{\Delta_{i}} \frac{|\Phi(x /|y|)|}{|y|^{n}}\left|\left(b(y)-b_{B_{i}}\right) f(y)\right| d y\right)^{q} d x \\
& +C_{q} \int_{\Delta_{k}}\left(\sum_{i=-\infty}^{k} \int_{\Delta_{i}} \frac{|\Phi(x /|y|)|}{|y|^{n}}(k-i)\|b\|_{\mathrm{CMO}^{1}\left(\mathbb{R}^{n}\right)}|f(y)| d y\right)^{q} d x \\
:= & I I_{1}+I I_{2} .
\end{aligned}
$$

For $I I_{1}$, by Lemma 3.1(i) and Hölder's inequality, we have

$$
\begin{aligned}
I I_{1} & \leq C_{q} A_{\Phi, r}^{q} \int_{\Delta_{k}}\left(\sum_{i=-\infty}^{k}|x|^{-n / r}\left(\int_{\Delta_{i}}\left|\left(b(y)-b_{B_{i}}\right) f(y)\right|^{r} d y\right)^{1 / r}\right)^{q} d x \\
& \leq C_{q} A_{\Phi, r}^{q} \int_{\Delta_{k}}\left(\sum_{i=-\infty}^{k}|x|^{-n / r}\left(\int_{\Delta_{i}}\left|\left(b(y)-b_{B_{i}}\right)\right|^{r q /(q-r)} d y\right)^{(q-r) / q r}\left(\int_{\Delta_{i}}|f(y)|^{q} d y\right)^{1 / q}\right)^{q} \\
& \leq C_{q} A_{\Phi, r}^{q}\left(\sum_{i=-\infty}^{k} 2^{k n(1 / q-1 / r)}\|b\|_{\mathrm{CMO}^{q r /(q-r)}\left(\mathbb{R}^{n}\right)}\left|B_{i}\right|^{(q-r) / q r}\left\|f X_{i}\right\|_{L^{q}}\right)^{q} \\
& =C_{q} A_{\Phi, r}^{q}\|b\|_{\mathrm{CMO}^{q r /(q-r)}\left(\mathbb{R}^{n}\right)}^{q}\left(\sum_{i=-\infty}^{k} 2^{(i-k) n(1 /)-1 / q)}\left\|f X_{i}\right\|_{L^{q}}\right)^{q} .
\end{aligned}
$$

For $I_{2}$, by Lemma 3.1(i), we have

$$
\begin{aligned}
I I_{2} & \leq C_{q} A_{\Phi, r}^{q}\|b\|_{\mathrm{CMO}^{1}\left(\mathbb{R}^{n}\right)}^{q} \int_{\Delta_{k}}\left(\sum_{i=-\infty}^{k}(k-i) 2^{i n(1 / r-1 / q)}|x|^{-n / r}\left\|f X_{i}\right\|_{L^{q}}\right)^{q} d x \\
& \leq C_{q} A_{\Phi, r}^{q}\|b\|_{\mathrm{CMO}^{1}\left(\mathbb{R}^{n}\right)}^{q} 2^{k n}\left(\sum_{i=-\infty}^{k}(k-i) 2^{i n(1 / r-1 / q)} 2^{-k n / r}\left\|f X_{i}\right\|_{L^{q}}\right)^{q} \\
& =C_{q} A_{\Phi, r}^{q}\|b\|_{\mathrm{CMO}^{1}\left(\mathbb{R}^{n}\right)}^{q}\left(\sum_{i=-\infty}^{k}(k-i) 2^{(i-k) n(1 / r-1 / q)}\left\|f X_{i}\right\|_{L^{q}}\right)^{q} .
\end{aligned}
$$


Following the estimates of $I, I I_{1}$, and $I I_{2}$, we can obtain that

$$
\begin{aligned}
\left\|H_{\Phi, b}^{1} f\right\|_{\dot{K}_{q}^{\alpha, p}\left(\mathbb{R}^{n}\right)} \leq & C A_{\Phi, r}\|b\|_{\mathrm{CMO}^{q}\left(\mathbb{R}^{n}\right)}\left(\sum_{k=-\infty}^{\infty} 2^{k \alpha p}\left(\sum_{i=-\infty}^{k} 2^{(i-k) n(1 / r-1 / q)}\left\|f X_{i}\right\|_{L^{q}}\right)^{p}\right)^{1 / p} \\
& +C A_{\Phi, r}\|b\|_{\mathrm{CMO}^{q r /(q-r)}\left(\mathbb{R}^{n}\right)}\left(\sum_{k=-\infty}^{\infty} 2^{k \alpha p}\left(\sum_{i=-\infty}^{k} 2^{(i-k) n(1 / r-1 / q)}\left\|f X_{i}\right\|_{L^{q}}\right)^{p}\right)^{1 / p} \\
& +C A_{\Phi, r}\|b\|_{\mathrm{CMO}^{1}\left(\mathbb{R}^{n}\right)}\left(\sum_{k=-\infty}^{\infty} 2^{k \alpha p}\left(\sum_{i=-\infty}^{k}(k-i) 2^{(i-k) n(1 / r-1 / q)}\left\|f X_{i}\right\|_{L^{q}}\right)^{p}\right)^{1 / p} \\
:= & S .
\end{aligned}
$$

Obviously,

$$
S \leq C A_{\Phi, r}\|b\|_{\mathrm{CMO}^{\max (q, q r /(q-r))}\left(\mathbb{R}^{n}\right)}\left(\sum_{k=-\infty}^{\infty} 2^{k \alpha p}\left(\sum_{i=-\infty}^{k}(k-i) 2^{(i-k) n(1 / r-1 / q)}\left\|f X_{i}\right\|_{L^{q}}\right)^{p}\right)^{1 / p} .
$$

When $0<p \leq 1$, we get

$$
\begin{aligned}
S^{p} & \leq C_{p} A_{\Phi, r}^{p}\|b\|_{\mathrm{CMO}^{\max (q, q r /(q-r))}\left(\mathbb{R}^{n}\right)}^{p} \sum_{k=-\infty}^{\infty} \sum_{i=-\infty}^{k} 2^{i \alpha p}\left\|f X_{i}\right\|_{L^{q}}^{p}(k-i)^{p} 2^{(i-k)(n / r-n / q-\alpha) p} \\
& =C_{p} A_{\Phi, r}^{p}\|b\|_{\mathrm{CMO}^{\max (q, q r /(q-r))}\left(\mathbb{R}^{n}\right)}^{p} \sum_{i=-\infty}^{\infty} 2^{i \alpha p}\left\|f X_{i}\right\|_{L^{q}}^{p} \sum_{k=i}^{\infty}(k-i)^{p} 2^{(i-k)(n / r-n / q-\alpha) p} \\
& =C_{p} A_{\Phi, r}^{p}\|b\|_{\mathrm{CMO}^{\max (q, q r /(q-r))}\left(\mathbb{R}^{n}\right)}^{p}\|f\|_{\dot{K}_{q}^{\alpha, p} .}^{p}
\end{aligned}
$$

For the case $p>1$, it follows from Hölder's inequality that

$$
\begin{aligned}
S^{p} \leq & C_{p} A_{\Phi, r}^{p}\|b\|_{\mathrm{CMO}^{\max (q, q r /(q-r))}\left(\mathbb{R}^{n}\right)}^{p} \sum_{k=-\infty}^{\infty} \sum_{i=-\infty}^{k} 2^{i \alpha p}\left\|f X_{i}\right\|_{L^{q}}^{p}{ }^{(i-k)(n / r-n / q-\alpha) p / 2} \\
& \times\left(\sum_{i=-\infty}^{k}(k-i)^{p^{\prime}} 2^{(i-k)(n / r-n / q-\alpha) p^{\prime} / 2}\right)^{p / p^{\prime}} \\
\leq & C_{p} A_{\Phi, r}^{p}\|b\|_{\mathrm{CMO}^{\max (q, q r /(q-r))}\left(\mathbb{R}^{n}\right)}^{p} \sum_{i=-\infty}^{\infty} 2^{i \alpha p}\left\|f X_{i}\right\|_{L^{q}}^{p} \sum_{k=i}^{\infty} 2^{(i-k)(n / r-n / q-\alpha) p / 2} \\
= & C_{p} A_{\Phi, r}^{p}\|b\|_{\mathrm{CMO}^{\max (q, q r /(q-r))}\left(\mathbb{R}^{n}\right)}^{p}\|f\|_{\dot{K}_{q}^{\alpha, p} .}^{p}
\end{aligned}
$$


When $\Phi$ satisfies (2.2) and $\alpha>-n\left(1 / q-1 / r^{\prime}\right)$, we get

$$
\begin{aligned}
\left\|\left(H_{\Phi, b}^{2} f\right) \chi_{k}\right\|_{L^{q}\left(\mathbb{R}^{n}\right)}^{q} \leq & C_{q} \int_{\Delta_{k}}\left(\sum_{i=k}^{\infty} \int_{\Delta_{i}} \frac{|\Phi(x /|y|)|}{|y|^{n}}\left|\left(b(x)-b_{B_{k}}\right) f(y)\right| d y\right)^{q} d x \\
& +C_{q} \int_{C_{k}}\left(\sum_{i=k}^{\infty} \int_{\Delta_{i}} \frac{|\Phi(x /|y|)|}{|y|^{n}}\left|\left(b(y)-b_{B_{k}}\right) f(y)\right| d y\right)^{q} d x \\
:=J+J J . &
\end{aligned}
$$

For $J$, using Lemma 3.1(ii), we have

$$
\begin{aligned}
J & \leq C_{q} B_{\Phi, r}^{q} \int_{\Delta_{k}}\left|b(x)-b_{B_{k}}\right|^{q}\left(\sum_{i=k}^{\infty} 2^{i n\left(1 / r^{\prime}-1 / q\right)}|x|^{-n / r^{\prime}}\left\|f X_{i}\right\|_{L^{q}}\right)^{q} d x \\
& \leq C_{q} B_{\Phi, r}^{q}\|b\|_{\mathrm{CMO}^{q}\left(\mathbb{R}^{n}\right)}^{q}\left|B_{k}\right|\left(\sum_{i=k}^{\infty} 2^{i n\left(1 / r^{\prime}-1 / q\right)} 2^{-k n / r}\left\|f X_{i}\right\|_{L^{q}}\right)^{q} \\
& =C_{q} B_{\Phi, r}^{q}\|b\|_{\mathrm{CMO}^{q}\left(\mathbb{R}^{n}\right)}^{q}\left(\sum_{i=k}^{\infty} 2^{(k-i) n\left(1 / q-1 / r^{\prime}\right)}\left\|f X_{i}\right\|_{L^{q}}\right)^{q} .
\end{aligned}
$$

For $J J$, by Lemma 3.2, we have

$$
\begin{aligned}
J J \leq & C_{q} \int_{\Delta_{k}}\left(\sum_{i=k}^{\infty} \int_{\Delta_{i}} \frac{|\Phi(x /|y|)|}{|y|^{n}}\left|\left(b(y)-b_{B_{i}}\right) f(y)\right| d y\right)^{q} d x \\
& +C_{q} \int_{\Delta_{k}}\left(\sum_{i=k}^{\infty} \int_{\Delta_{i}} \frac{|\Phi(x /|y|)|}{|y|^{n}}(i-k)\|b\|_{\mathrm{CMO}^{1}\left(\mathbb{R}^{n}\right)}|f(y)| d y\right)^{q} d x \\
:= & J J_{1}+J J_{2} .
\end{aligned}
$$

For $J J_{1}$, by Lemma 3.1(ii) and Hölder's inequality, similarly to $I I_{1}$, we have

$$
J J_{1} \leq C_{q} B_{\Phi, r}^{q}\|b\|_{\mathrm{CMO}^{q r(q-r)}\left(\mathbb{R}^{n}\right)}^{q}\left(\sum_{i=k}^{\infty} 2^{(k-i) n\left(1 / q-1 / r^{\prime}\right)}\left\|f X_{i}\right\|_{L^{q}}\right)^{q} .
$$

For $J_{2}$, also due to Lemma 3.1(ii), we have

$$
\begin{aligned}
J J_{2} & \leq C_{q} B_{\Phi, r}^{q}\|b\|_{\mathrm{CMO}^{1}\left(\mathbb{R}^{n}\right)}^{q} \int_{\Delta_{k}}\left(\sum_{i=k}^{\infty}(i-k) 2^{i n\left(1 / r^{\prime}-1 / q\right)}|x|^{-n / r^{\prime}}\left\|f X_{i}\right\|_{L^{q}}\right)^{q} d x \\
& \leq C_{q} B_{\Phi, r}^{q}\|b\|_{\mathrm{CMO}^{1}\left(\mathbb{R}^{n}\right)}^{q} 2^{k n}\left(\sum_{i=k}^{\infty}(i-k) 2^{i n\left(1 / r^{\prime}-1 / q\right)} 2^{-k n / r^{\prime}}\left\|f X_{i}\right\|_{L^{q}}\right)^{q} \\
& =C_{q} B_{\Phi, r}^{q}\|b\|_{\mathrm{CMO}^{1}\left(\mathbb{R}^{n}\right)}^{q}\left(\sum_{i=k}^{\infty}(i-k) 2^{(k-i) n\left(1 / q-1 / r^{\prime}\right)}\left\|f X_{i}\right\|_{L^{q}}\right)^{q} .
\end{aligned}
$$

The remaining proof is similar to the proof of (a), so that of (b) can be obtained easily. 
(c) When $\Phi$ satisfies (2.1), (2.2), and $-n\left(1 / q-1 / r^{\prime}\right)<\alpha<n(1 / r-1 / q)$, due to

$$
H_{\Phi, b} f(x)=H_{\Phi, b}^{1} f(x)+H_{\Phi, b}^{2} f(x)
$$

therefore, by (a) and (b), we can easily get (c).

Proof of Theorem 2.8. (a) When $\Phi$ satisfies (2.1) and $\alpha<n\left(1 / r-1 / q_{1}\right)$, by Lemmas 3.3, 3.1(i), and $1 / q_{1}-1 / q_{2}=r / n$, we get

$$
\begin{aligned}
\left\|\left(H_{\Phi, b}^{1} f\right) X_{k}\right\|_{L^{q_{2}}}^{q_{2}} & \leq\|b\|_{\Lambda_{r}}^{q_{2}} \int_{\Delta_{k}}\left(\sum_{i=-\infty}^{k} \int_{\Delta_{i}} \frac{|\Phi(x /|y|)|}{|y|^{n}}\left(|x|^{\gamma}+|y|^{\gamma}\right)|f(y)| d y\right)^{q_{2}} d x \\
& \leq C\|b\|_{\Lambda_{r}}^{q_{2}} \int_{\Delta_{k}}\left(|x|^{\gamma} \sum_{i=-\infty}^{k} \int_{\Delta_{i}} \frac{|\Phi(x /|y|)|}{|y|^{n}}|f(y)| d y\right)^{q_{2}} d x \\
& \leq C_{q_{2}} A_{\Phi, r}^{q_{2}}\|b\|_{\Lambda_{r}}^{q_{2}} 2^{k n}\left(2^{k \gamma-k n / r} \sum_{i=-\infty}^{k} 2^{i n\left(1 / r-1 / q_{1}\right)}\left\|f X_{i}\right\|_{L^{q_{1}}}\right)^{q_{2}} \\
& =C_{q_{2}} A_{\Phi, r}^{q_{2}}\|b\|_{\Lambda_{r}}^{q_{2}}\left(\sum_{i=-\infty}^{k} 2^{(i-k) n\left(1 / r-1 / q_{1}\right)}\left\|f X_{i}\right\|_{L^{q_{1}}}\right)^{q_{2}} .
\end{aligned}
$$

The remaining proof Is similar to the proof of (a) of Theorem 2.6, so that of (a) can be obtained easily.

(b) When $\Phi$ satisfies (2.2) and $\alpha>-n\left(1 / q_{2}-1 / r^{\prime}\right)$, by Lemmas 3.3, 3.1(ii), and $\left(1 / q_{1}\right)-$ $\left(1 / q_{2}\right)=\gamma / n$, we get

$$
\begin{aligned}
\left\|\left(H_{\Phi, b}^{2} f\right)_{X k}\right\|_{L^{q_{2}\left(\mathbb{R}^{n}\right)}}^{q_{2}} & \leq\|b\|_{\Lambda_{r}}^{q_{2}} \int_{\Delta_{k}}\left(\sum_{i=k}^{\infty} \int_{\Delta_{i}} \frac{|\Phi(x /|y|)|}{|y|^{n}}\left(|x|^{\gamma}+|y|^{\gamma}\right)|f(y)| d y\right)^{q_{2}} d x \\
& \leq C_{q_{2}}\|b\|_{\Lambda_{r}}^{q_{2}} \int_{\Delta_{k}}\left(\sum_{i=k}^{\infty} \int_{\Delta_{i}}|y|^{r} \frac{|\Phi(x /|y|)|}{|y|^{n}}|f(y)| d y\right)^{q_{2}} d x \\
& \leq C_{q_{2}} B_{\Phi, r}^{q_{2}}\|b\|_{\Lambda_{r}}^{q_{2}} \int_{\Delta_{k}}\left(\sum_{i=k}^{\infty} 2^{i r} 2^{i n\left(1 / r^{\prime}-1 / q_{1}\right)}|x|^{-n / r^{\prime}}\left\|f X_{i}\right\|_{L^{q_{1}}}\right)^{q_{2}} d x \\
& \leq C_{q_{2}} B_{\Phi, r}^{q_{2}}\|b\|_{\Lambda_{r}}^{q_{2}}\left(\sum_{i=k}^{\infty} 2^{(k-i) n\left(1 / q_{2}-1 / r^{\prime}\right)}\left\|f X_{i}\right\|_{L^{q_{1}}}\right)^{q_{2}} .
\end{aligned}
$$

The remaining proof is similar to the proof of (a) of Theorem 2.6, so that of (b) can be obtained easily.

(c) The proof is the same as the proof of (c) of Theorem 2.6. 
Proof of Theorem 2.10. We only give the proof of (a) when $\lambda>0$. The proof of (b) is similar to that for (a), and the proof of (c) is similar to that for (c) of Theorem 2.6. By the definition of Morrey-Herz spaces and the estimates for $I, I I_{1}$, and $I I_{2}$ above, we have

$$
\begin{aligned}
\| & H_{\Phi, b}^{1} f \|_{M \dot{K}_{p, q}^{\alpha, \lambda}} \\
\leq & C A_{\Phi, r}\|b\|_{C M O^{q}\left(\mathbb{R}^{n}\right)} \sup _{k_{0} \in \mathbb{Z}} 2^{-k_{0} \lambda}\left(\sum_{k=-\infty}^{k_{0}} 2^{k \alpha p}\left(\sum_{i=-\infty}^{k} 2^{(i-k)(n / r-n / q)}\left\|f X_{i}\right\|_{L^{q}}\right)^{p}\right)^{1 / p} \\
& +C A_{\Phi, r}\|b\|_{C \mathrm{CMO}^{q r /(q-r)}\left(\mathbb{R}^{n)}\right)} \sup _{k_{0} \in \mathbb{Z}} 2^{-k_{0} \lambda}\left(\sum_{k=-\infty}^{k_{0}} 2^{k \alpha p}\left(\sum_{i=-\infty}^{k} 2^{(i-k)(n / r-n / q)}\left\|f X_{i}\right\|_{L^{q}}\right)^{p}\right)^{1 / p} \\
& +C A_{\Phi, r}\|b\|_{\mathrm{CMO}^{1}\left(\mathbb{R}^{n}\right)} \sup _{k_{0} \in \mathbb{Z}} 2^{-k_{0} \lambda}\left(\sum_{k=-\infty}^{k_{0}} 2^{k \alpha p}\left(\sum_{i=-\infty}^{k}(k-i) 2^{(i-k)(n / r-n / q)}\left\|f X_{i}\right\|_{L^{q}}\right)^{p}\right)^{1 / p} \\
:= & E_{1}+E_{2}+E_{3} .
\end{aligned}
$$

For $E_{1}$, noting that $\alpha<n(1 / r-1 / q)+\lambda$, we have

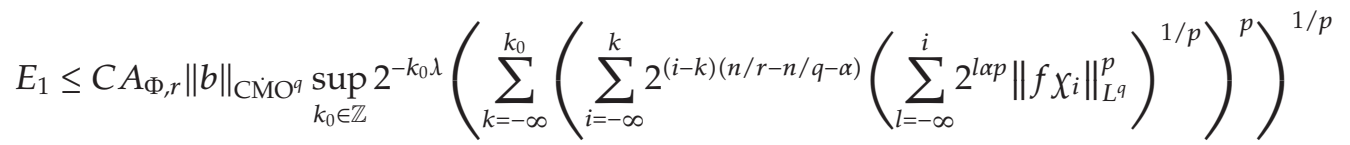

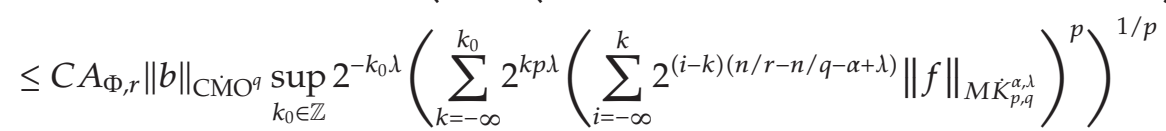

$$
\begin{aligned}
& \leq C A_{\Phi, r}\|b\|_{C \mathrm{CMO}^{q}} \sup _{k_{0} \in \mathbb{Z}} 2^{-k_{0} \lambda}\left(\sum_{k=-\infty}^{k_{0}} 2^{k p \lambda}\right)^{1 / p}\|f\|_{M \dot{K}_{p, q}^{\alpha, \lambda}} \\
& =C A_{\Phi, r}\|b\|_{\mathrm{CMO}^{q}\left(\mathbb{R}^{n}\right)}\|f\|_{M \dot{K}_{p, q}^{\alpha, \lambda}\left(\mathbb{R}^{n}\right)} \text {. }
\end{aligned}
$$

Similarly to the proof of $E_{1}$, we have

$$
\begin{gathered}
E_{2} \leq C A_{\Phi, r}\|b\|_{\mathrm{CMO}^{q r /(q-r)}\left(\mathbb{R}^{n}\right)}\|f\|_{M \dot{K}_{p, q}^{\alpha, \lambda}\left(\mathbb{R}^{n}\right)^{\prime}} \\
E_{3} \leq C A_{\Phi, r}\|b\|_{\mathrm{CMO}^{1}\left(\mathbb{R}^{n}\right)}\|f\|_{M \dot{K}_{p, q}^{\alpha, \lambda}\left(\mathbb{R}^{n}\right)} .
\end{gathered}
$$

This finishes the proof of Theorem 2.10.

Proof of Theorem 2.11. The proof is similar to the proof of Theorem 2.10, so we omit the details.

\section{Acknowledgment}

The authors are supported in part by the NSF of China, no: 10931001, 10871173. 


\section{References}

[1] A. K. Lerner and E. Liflyand, "Multidimensional Hausdorff operators on the real Hardy space," Journal of the Australian Mathematical Society, vol. 83, no. 1, pp. 79-86, 2007.

[2] E. Liflyand and A. Miyachi, "Boundedness of the Hausdorff operators in $H^{p}$ spaces, $0<P<1$," Studia Mathematica, vol. 194, no. 3, pp. 279-292, 2009.

[3] E. Liflyand and F. Moricz, "The Hausdorff operators is bounded on real $H^{1}$ space," Proceedings of the American Mathematical Society, vol. 128, pp. 1391-1396, 2000.

[4] J. C. Chen, D. S. Fan, and J. Li, "Hausdorff operators on function spaces," Chinese Annals of Mathematics B. In press.

[5] M. J. Carro and J. Soria, "Weighted Lorentz spaces and the Hardy operator," Journal of Functional Analysis, vol. 112, no. 2, pp. 480-494, 1993.

[6] M. Christ and L. Grafakos, "Best constants for two nonconvolution inequalities," Proceedings of the American Mathematical Society, vol. 123, no. 6, pp. 1687-1693, 1995.

[7] S. C. Long and J. Wang, "Commutators of Hardy operators," Journal of Mathematical Analysis and Applications, vol. 274, no. 2, pp. 626-644, 2002.

[8] E. Sawyer, "Weighted Lebesgue and Lorentz norm inequalities for the Hardy operator," Transactions of the American Mathematical Society, vol. 281, no. 1, pp. 329-337, 1984.

[9] Z. W. Fu, Z. G. Liu, S. Z. Lu, and H. B. Wang, "Characterization for commutators of $n$-dimensional fractional Hardy operators," Science in China A, vol. 50, no. 10, pp. 1418-1426, 2007.

[10] J. C. Chen and G. L. Gao, "Commutators of Hausdorff operator," Submitted.

[11] S. Lu and D. Yang, "The central BMO spaces and Littlewood-Paley operators," Approximation Theory and its Applications. New Series, vol. 11, no. 3, pp. 72-94, 1995.

[12] M. Paluszyński, "Characterization of the Besov spaces via the commutator operator of Coifman, Rochberg and Weiss," Indiana University Mathematics Journal, vol. 44, no. 1, pp. 1-18, 1995.

[13] S. Z. Lu, D. C. Yang, and G. E. Hu, "Herz Type Spaces and Their Application," pp. Science PressBeijing, China, 2008.

[14] S. Lu, D. Yang, and Z. Zhou, "Sublinear operators with rough kernel on generalized Morrey spaces," Hokkaido Mathematical Journal, vol. 27, no. 1, pp. 219-232, 1998.

[15] Q.-Y. Zheng and Z.-W. Fu, "Lipschitz estimates for commutators of N-dimensional fractional Hardy operators," Communications in Mathematical Research, vol. 25, no. 3, pp. 241-245, 2009. 


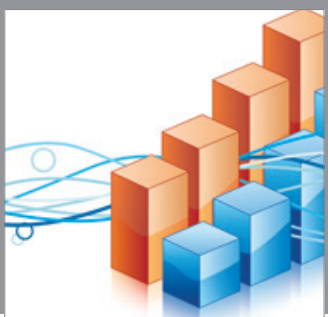

Advances in

Operations Research

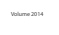

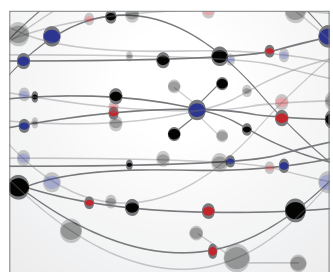

\section{The Scientific} World Journal
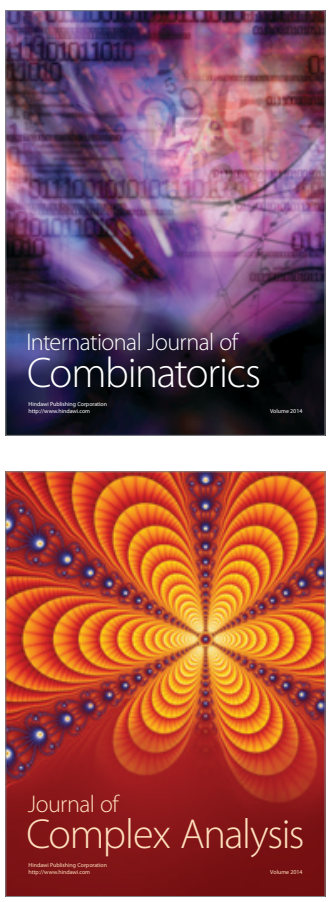

International Journal of

Mathematics and

Mathematical

Sciences
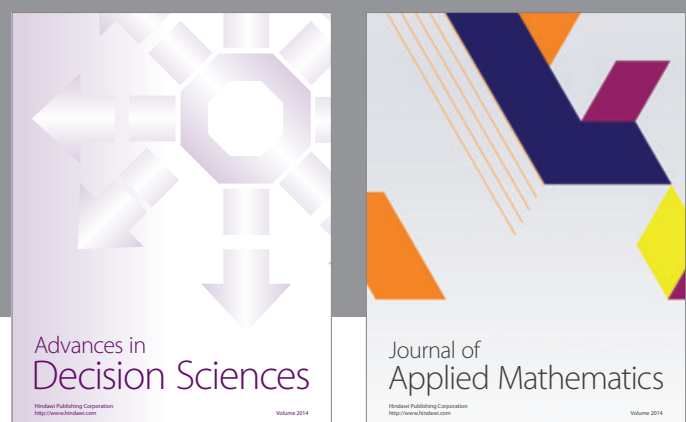

Journal of

Applied Mathematics
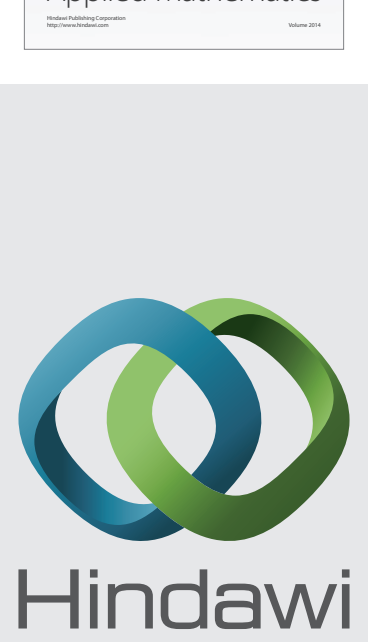

Submit your manuscripts at http://www.hindawi.com
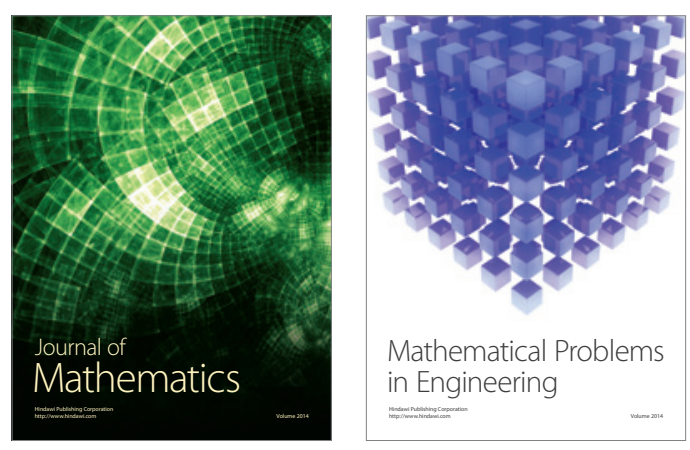

Mathematical Problems in Engineering
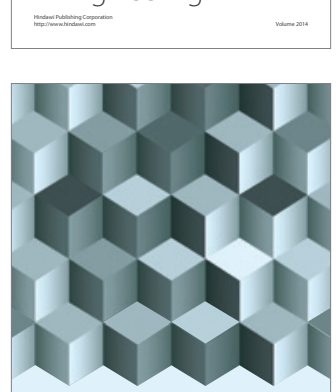

Journal of

Function Spaces
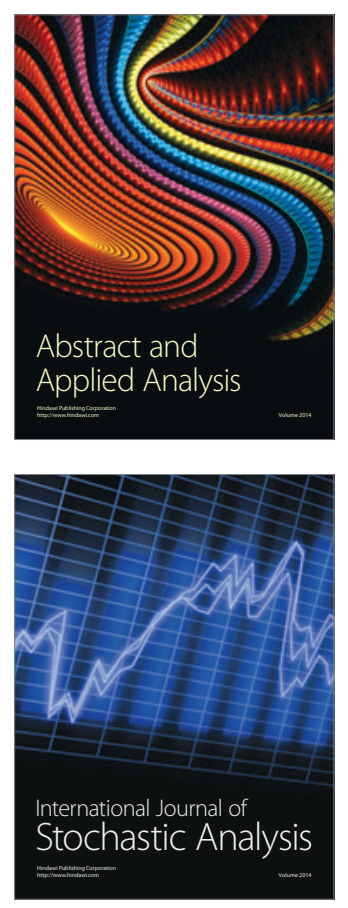

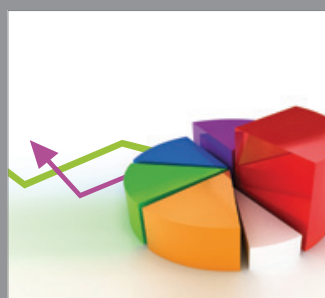

ournal of

Probability and Statistics

Promensencen
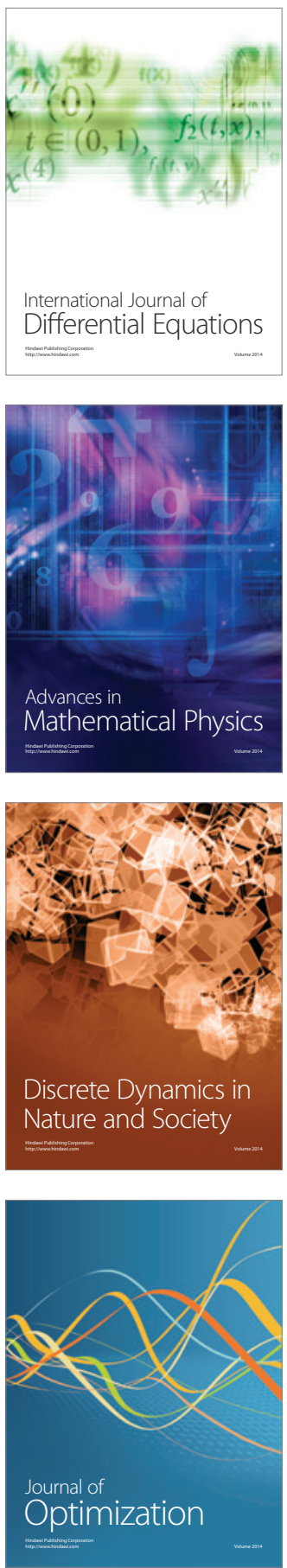